\title{
Using planetary transits to estimate magnetic cycles lengths in Kepler stars
}

\author{
Raissa Estrela and Adriana Valio \\ Center for Radio Astronomy and Astrophysics (CRAAM), Mackenzie Presbyterian University, \\ Sao Paulo, Brazil \\ Rua da Consolacao 01301-000, 896, Sao Paulo, Brazil \\ email: rlf.estrela@gmail.com \\ email: avalio@craam.mackenzie.br
}

\begin{abstract}
Observations of various solar-type stars along decades showed that they could have magnetic cycles, just like our Sun. These observations yield a relation between the rotation period $P_{r o t}$ and the cycle length $P_{c y c l e}$ of these stars. Two distinct branches for the cycling stars were identified: active and inactive, classified according to stellar activity level and rotation rate. In this work, we determined the magnetic activity cycle for 6 active stars observed by the Kepler telescope. The method adopted here estimates the activity from the excess in the residuals of the transit light curves. This excess is obtained by subtracting a spotless model transit from the light curve, and then integrating over all the residuals during the transit. The presence of long term periodicity is estimated from the analysis of a Lomb-Scargle periodogram of the complete time series. Finally, we investigate the rotation-cycle period relation for the stars analysed here.
\end{abstract}

Keywords. magnetic, activity, rotation.

\section{Introduction}

The magnetic activity of the Sun varies throughout the 22 year long magnetic cycle, with the polarity of its magnetic field flipping every 11 years. These cycles are identified by the frequency and number of sunspots in the solar surface, which acts as an indicator of activity. Stellar activity is also present in other stars, that show remarkable lightcurve variations due to starspots and other magnetic phenomena. Skumanich (1972) first suggested that the activity of the star was associated with its rotation rate, and consequently with it age. Therefore, young rapidly rotating stars show higher level of activity and can produce larger spots and energetic flares.

The Mount Wilson Observatory Ca II H K survey was the first to show that hundred of stars could also exhibit long and short periodic cycles between 2.5 and 25 years (Baliunas et al., 1995). Using this data, Saar \& Brandenburg (1999) established a relation between the stellar rotation period, $\mathrm{P}_{\text {orb }}$ and stellar cycle period, $\mathrm{P}_{\text {cycle }}$ as a function of the Rossby number. This relation divided the stars in two branches: active (A) and inactive (I), according to its activity level and rotation rate. The active sequence is composed by stars that rotates faster than the Sun, while the inactive one has slow rotating stars. Later, it was observed that some of the stars in the active branch also exhibits secondary short cycles that fall in the inactive branch (Böhm-Vitense, 2007).

Recently, the Kepler telescope provided long-term high photometric precision of thousand of stars. These data offer an unique opportunity to increase our understanding about magnetic cycles in other stars. In addition, it could improve stellar dynamo models in different type of stars. Among the studies of magnetic cyles using Kepler data, Vida \& Olah (2013) analyzed fast-rotating stars and found activity cycles of 300-900 days for 9 
Table 1.

Observational parameters of the stars

\begin{tabular}{lccccl} 
& Radius $\left.\left[\mathrm{R}_{\odot}\right\}\right]$ & Age $[\mathrm{Gyr}]$ & $\begin{array}{l}\text { Effective } \\
\text { Temperature }[\mathrm{K}]\end{array}$ & $\begin{array}{l}\text { Rotation } \\
\text { Period [days] }\end{array}$ & Reference \\
\hline Kepler-17 & $1.05 \pm 0.03$ & $<1.78$ & $5780 \pm 80$ & 11.89 & 1,2 \\
Kepler-63 & $0.901_{-0.022}^{+0.022}$ & 0.2 & $5580 \pm 50$ & 5.40 & 3 \\
KIC 9705459 & $0.951_{-0.04}^{+0.159}$ & & $5900_{-125}^{+106}$ & 2.83 & 4,5 \\
KIC 5376836 & $0.885_{-0.081}^{+0.363}$ & & $5903_{-112}^{+93}$ & $\sim 4$ & 4,5 \\
Kepler-96 & $1.02 \pm 0.09$ & 2.34 & $5690.0 \pm 73.0$ & 15.30 & 5,6 \\
Hat-p-11 (Kepler-3) & $0.75 \pm 0.02$ & $6.5_{-4.1}^{+4.1}$ & $4780.0 \pm 50.0$ & 30 & 7 \\
\hline
\end{tabular}

${ }^{1}$ References. (1) Bonomo et al. (2012), (2) Désert et al. (2011), (3) Sanchis-Ojeda et al. (2013), (4) MAST Kepler database, (5) Walkowicz \& Basri (2013), (6) Marcy et al. (2014) and (7) Sanchis-Ojeda \& Winn (2011).

targets, and Marthur et al. (2014) found evidences of magnetic cycle in two Kepler solar type star.

\section{Spot model}

Kepler-17 and Kepler-63 are two active solar-type stars that exhibits rotational modulations in their light curve caused by the presence of starspots, with a peak-to-peak variation of $6 \%$ and $4 \%$, respectively. To analyze and characterize the physical parameters of the spots in these stars, we applied the transit model proposed by Silva (2003). This model simulates the passage of a planet in front of its host star. The simulation consider a star with quadratic limb darkening as a 2D image and the planet is assumed to be a dark disk with radius $R_{p} / R_{\text {star }}$, where $R_{p}$ is the radius of the planet and $R_{\text {star }}$ is the radius of the primary star. The sum of all the pixels in the image (star plus dark planet) yields the transit light curve.

We added round spots to the stellar surface and modelled them by three parameters: intensity (in function of the intensity at disc center $I_{c}$ ), radius (measured in units of planetary radius) and position (longitude and latitude). The latitude remains fixed and equal to the transit latitude. The longitude of the spots is limited to -70 and $70^{\circ}$ from the central meridian to avoid any distortions caused by the ingress and the egress of the transit. In the case of Kepler-17, the latitude is $-14^{\circ} .6$, while for Kepler- $63 \mathrm{~b}$ the planet occults several latitudes of the star from its equator all the way to the poles due to its high obliquity.

To obtain a better fit for each transit light curve, it was necessary to refine the values for the semi-major axis and planet radius obtained from literature for Kepler-17b (Désert et al., 2011) and for Kepler-63b (Sanchis-Ojeda et al., 2013). The physical parameters of the stars are described in Table 1.

To fit the spots, we subtracted a spotless model from the transit light curve. The result of this subtraction are the residuals, where the spots became more evident, as the "bumps" seen in the residuals. This process is illustrated in Fig. 1c for Kepler-17. We used the CDPP (Combined Differential Photometric Precision, see Christiansen et al. (2012) as an estimation of the noise in the Kepler data. Each quarter of the light curve has an associated CDPP value. Here, we considered the uncertainty in the data, $\sigma$, as being ten times the average of the CDPP values in all quarters. Only the "bumps" that exceed the detection limit of $10 \sigma$ are assumed as spots and modelled. Finally, the best fit of the spots parameters is obtained by minimizing $\chi^{2}$, calculated using the AMOEBA routine (Press et al., 1992). 


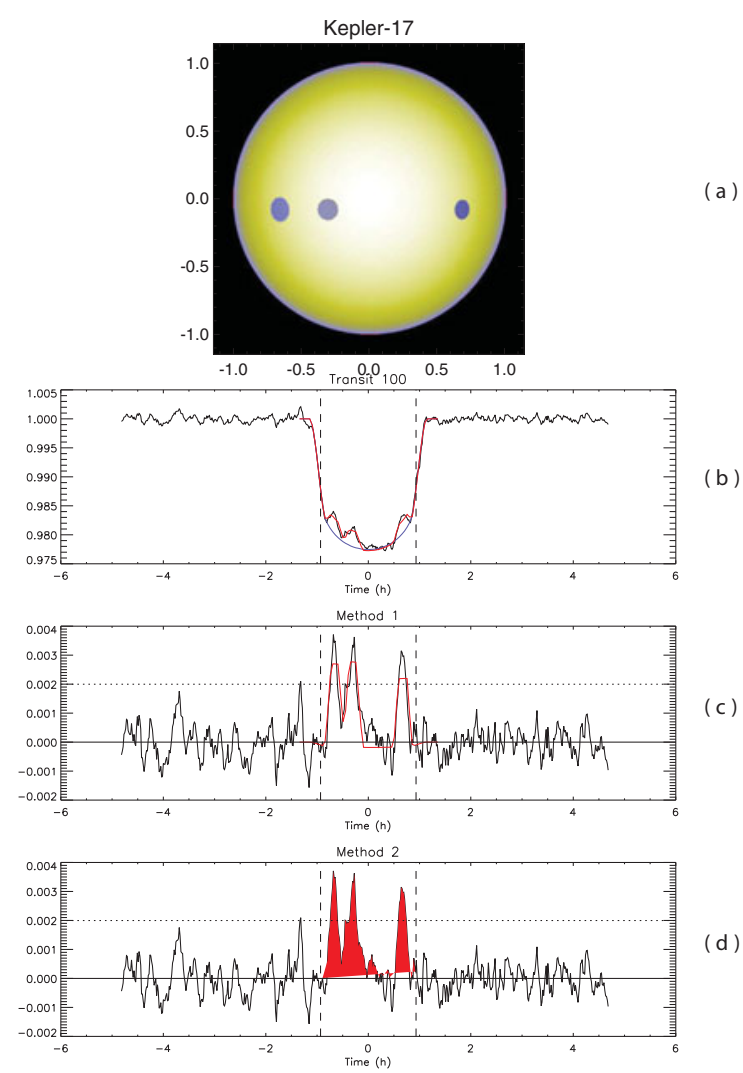

Figure 1. The 100th transit from Kepler-17 illustrates a typical example of the two methods adopted in this work: the spot model developed by Silva (2003) and transit residual excess. (a): Synthetised star with three spots. (b): Transit light curve with the model of a spotless star overplotted (red). (c) Residuals of the transit lightcurve after subtraction of a spotless star model. The red curve shows the fit to the data "bumps". (d): Integration (in red) of the residual excess resulted from the subtraction.

The determination of the stellar cycles was performed by using two approaches (Estrela $\&$ Valio (2016)). The first one is the analysis of the variation in the number of spots during the 4 years of observation of the Kepler stars. The latter is the calculation of the flux deficit resulting from the presence of spots on the star surface. The relative flux deficit of a single spot is the product of the spot contrast and its area, thus for each transit the total flux deficit associated with spots was calculated by summing all individuals spots:

$$
F \approx \sum\left(1-f_{i}\right)\left(R_{\text {spot }}\right)^{2}
$$

where the spots contrast is taken to be $\left(1-f_{i}\right)$, and $f_{i}$ is the relative intensity of the spot with respect to the disk center intensity $I_{c}$. A value of $f_{i}=1$ means that there is no spot at all.

Possible long duration trends were removed in these time series by applying a quadratic polynomial fit and then subtracting it. Then, a Lomb Scargle periodogram (LS) (Scargle, 1982) was applied on these time series to obtain the period related to the magnetic cycle. In addition, it was applied a significance test to quantify the significance of the peaks from the LS periodogram. The statistical significance associated to each frequency in the periodogram is determined by the $\mathrm{p}$-value $(\mathrm{p})$. The smaller the $\mathrm{p}$-value, the larger the significance of the peak. We adopted the significance level $\alpha$ as being $3 \sigma(\mathrm{p} \pm 0.0013)$. 

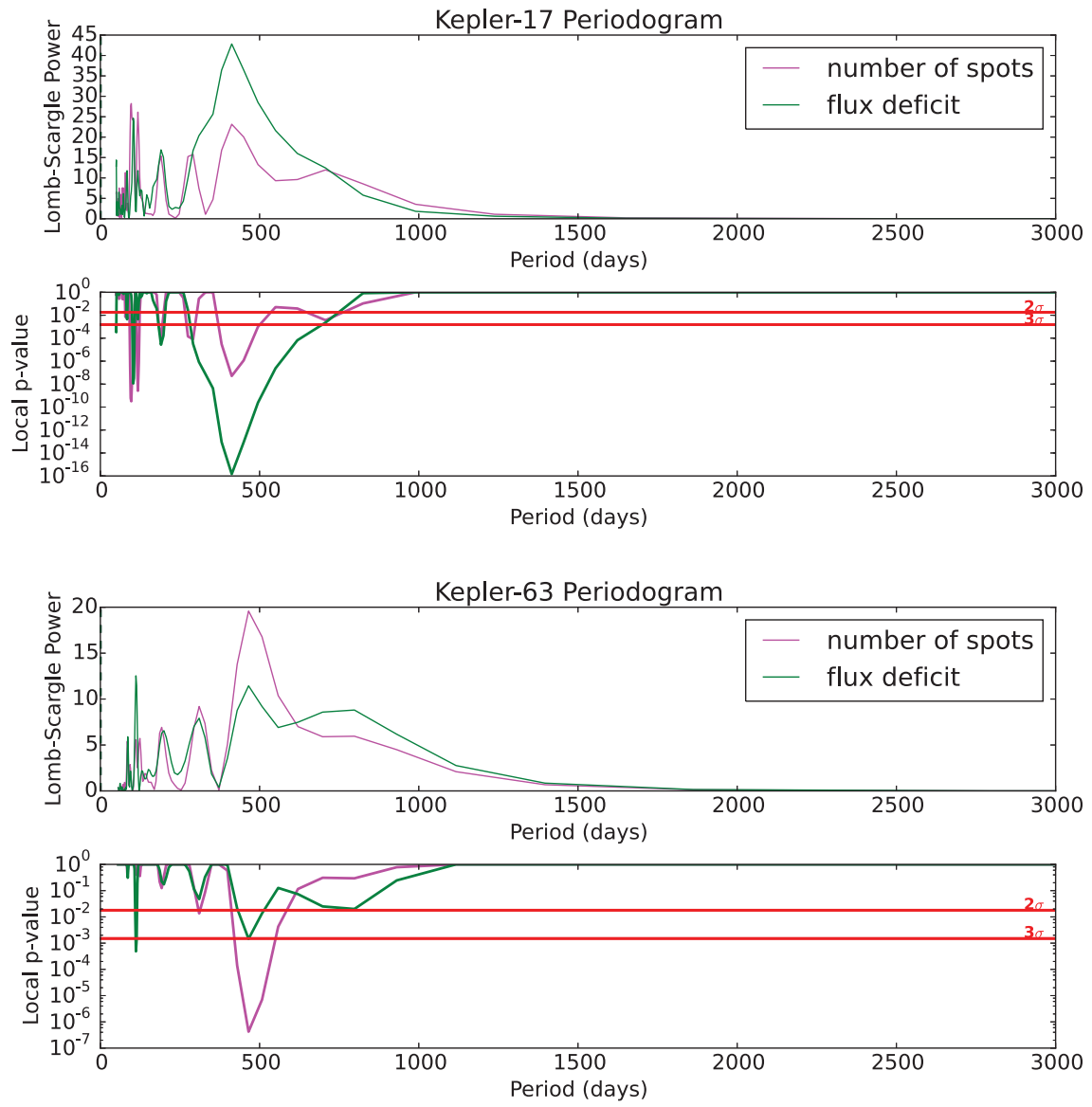

Figure 2. Lomb Scargle periodogram applied to the number of spots and total flux deficit of Kepler-17 (top) and Kepler-63 (bottom). The highest peak, indicated by a dashed line, corresponds to a periodicity of $410 \pm 50$ days for Kepler- 17 and $460 \pm 60$ days for Kepler- 63.

Each periodogram in Figure 2 has a significance test associated (plotted below). The uncertainty of the peaks in the periodogram is given by the FWHM of the peak power.

The LS periodogram detected a long term periodicity for both stars, as shown in Fig. 2 . Kepler-17 shows a prominent peak at $410 \pm 60$ days (number of spots) and $410 \pm 50$ days (flux deficit), while Kepler-63 shows a periodicity of $460 \pm 60$ days for the total number of spots, and $460 \pm 50$ days for the flux deficit. Detailed analysis of the results using the spot modeling for these two stars are described in Estrela \& Valio (2016).

\section{Transit residuals excess}

In the second method of this work we subtracted a modelled light curve of a star without spots from the transit light curves. The result from this subtraction is the residual that clearly shows the spots signatures. An example of this method is shown in Figure 1 for the 100th transit of Kepler-17. The excess in the residuals (in bold in Fig. 1d) corresponds to the spots signatures. Thus, we integrated the residual excess constrained to $\pm 70^{\circ}$ longitude of the star (delimited by the vertical dashed lines of Fig.1). This 

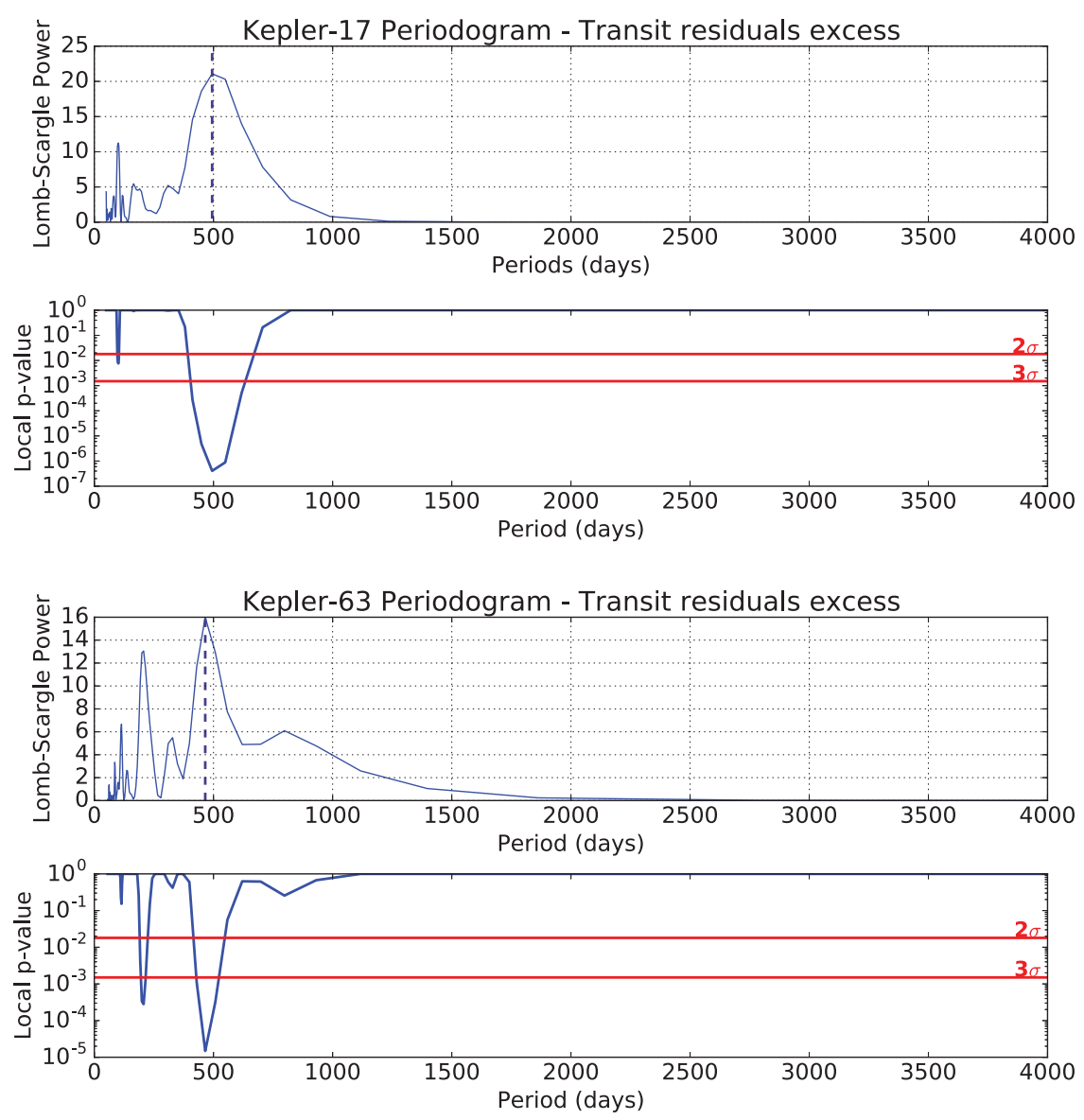

Figure 3. Lomb Scargle periodogram applied to the integrated transit residuals excess of Kepler-17 (top) and Kepler-63 (bottom). The highest peak, indicated by a dashed line, corresponds to a periodicity of $494 \pm 100$ days for Kepler-17 and $465 \pm 40$ days for Kepler-63.

allows us to characterize the magnetic activity level of the star. To remove any possible trends in this time series, we applied a quadratic polynomial fit and subtracted.

We applied the LS periodogram to the time series of integrated transit residuals excess. A peak at $490 \pm 100$ days was found for Kepler-17 and $460 \pm 40$ days for Kepler-63. The value obtained for Kepler-63 is similar to that from the first approach, and corresponds to a 1.27 year-cycle. On the other hand, the cycle period estimate for Kepler-17 agree within the uncertainty of the result from the first method.

Therefore, the results of both methods agree with each other. For this reason, we decided to work with more stars applying only the second method (transit residuals excess), which is easier in estimating magnetic cycles.

This method was applied to four more Kepler active stars hosting planets: Hat-p-11 (Kepler-3), Kepler-96, KIC 9705459 and KIC 5376836 (see details in Table 1). However, the planets candidates orbiting KIC 9705459 and KIC 5376836 were later classified as false positive, and the two stars are now known to be a binary eclipsing system. In this case, the transit of a companion star can also occult a spot in the stellar disk and show its signature, which works similar when eclipsed by a planetary transit. For this reason, we decided to keep these stars in our analysis. Then, by applying a Lomb Scargle periodogram, we found a clean peak at $305 \pm 60$ days for Hat-p-11. Kepler-96 and KIC 
Table 2.

Magnetic activity cycle periods

\begin{tabular}{lcc}
\hline Star & $\mathrm{P}_{\text {cycle }}$ (days) & $\mathrm{P}_{\text {cycle }}$ (years) \\
Kepler-17 & $490 \pm 100$ & $1.35 \pm 0.27 \mathrm{yr}$ \\
Kepler-63 & $460 \pm 60$ & $1.27 \pm 0.16 \mathrm{yr}$ \\
Hat-p-11 (Kepler-3) & $305 \pm 60$ & $0.83 \pm 0.16$ \\
Kepler-96 & $545 \pm 128$ & $1.50 \pm 0.35$ \\
KIC 9705459 & $100 \pm 9$ & $0.27 \pm 0.024$ \\
KIC 5376836 & $42 \pm 3$ & $0.11 \pm 0.007$ \\
\hline
\end{tabular}

9705459 also showed a clean peak at $545 \pm 128$ days and $100 \pm 9$ days, respectively. Finally, KIC 5376836 showed a significant peak at $42 \pm 3$ days. These periodicities show a p-value below the $3 \sigma$ significance level, confirming their significance. Table 2 shows a summary of the results found for the magnetic cycles of all stars analysed in this work.

\section{Summary and Conclusions}

We have estimated the period of the magnetic cycle, $\mathrm{P}_{\text {cycle }}$, for two active solar-type stars, Kepler-17 and Kepler-63, by applying two new methods: spot modelling and transit residuals excess. Since the results of both methods agreed with each other, we used the second method to estimate the magnetic cycle of four more active stars observed by Kepler: Hat-p-11 (Kepler-3), Kepler-96, KIC 9705459 and KIC 5376836. This method is much faster to determine magnetic cycles because it only requires to integrate the area of the residuals due to the activity (spots) in the transit light curve. The first two stars have a transiting planet, while KIC 9705459 and KIC 5376836 had planet candidates, found later to be false positives. These stars were classified as eclipsing binary systems, and we used the primary transit in our analysis to detect spots.

The results found here, with an exception of KIC 5376836 and KIC 9705459, have a $\mathrm{P}_{\text {cycle }}$ within the same range of 300-900 days found for the 9 Kepler fast-rotators stars analyzed by Vida \& Olah (2013). As we are constrained to the duration of observation $(\leqslant 4$ years) of the Kepler telescope, it is not possible to determine longer cycles.

Based on the relation between the stellar rotation period, $\mathrm{P}_{\text {orb }}$ and stellar cycle period, $\mathrm{P}_{\text {cycle }}$, proposed by Saar \& Brandenburg (1999), we can verify that Kepler-63, KIC 5376836 and KIC 9705459 fall within the active branch. Kepler-96 and Kepler-17 have their short cycles in the inactive branch, however, as proposed by Böhm-Vitense (2007) stars, the active branch may also shows secondary cycles that fall in the inactive branch. Finally, Hat-p-11 is a special case compared to the other stars in our sample. This star has a long rotation period ( $\mathrm{P}_{\text {rot }} \sim 30$ days) and has an age older than the Sun (6.5 Gyrs), but it shows remarkable activity. Probably, Hat-p-11 is interacting magnetically with its close orbit Hot-Jupiter, and for this reason its activity level is higher. The short cycle found for this star falls close to the inactive branch.

\section{References}

Baliunas, S. L., Donahue, R. A., Soon, W. H., et al. 1995, ApJ, 438, 269

Böhm-Vitense, E. 2007, ApJ, 657, 486

Bonomo, A. S., Hébrard, G., Santerne, A., et al. 2012, A\&SA, 538, A96

Christiansen, J. L., Jenkins, J. M., Caldwell, D. A., et al. 2012, PASP, 124, 1279

Désert, J.-M., Charbonneau, D., Demory, B.-O., et al. 2011, ApJS, 197, 14

Estrela, R. \& Valio, A. 2016, ApJ, 831, 57 
Marcy, G. W., Isaacson, H., Howard, A. W., et al. 2014, ApJS, 210, 20

Mathur, S., García, R. A., Ballot, J., Ceillier, T., Salabert, D., Metcalfe, T. S., Régulo, C., Jiménez, A., \& Bloemen, S. 2014, A\& A, 562A, 124M

Press, W. H., Teukolsky, S. A., Vetterling, W. T., \& Flannery, B. P. 1992, Cambridge: University Press, - c1992, 2nd ed.,

Saar, S. H. \& Brandenburg, A. 1999, ApJ, 524, 295

Sanchis-Ojeda, R. \& Winn, J. N. 2011, ApJ, 743, 61

Sanchis-Ojeda, R., Winn, J. N., Marcy, G. W., et al. 2013, ApJ, 775, 54

Scargle, J. D. 1982, ApJ, 263, 835

Silva, A. V. R. 2003, Ap. Lett., 585, L147

Skumanich, A. 1972, ApJ, 171, 565S

Vida, K. \& Olah, R. 2013, MNRS, 441, 2744V

Walkowicz, L. M. \& Basri, G. S. 2013, MNRAS, 436, 1883 